\title{
Editorial
}

\section{Inverse Problems: Theory and Application to Science and Engineering 2015}

\author{
Davide La Torre, ${ }^{1,2}$ Herb Kunze, ${ }^{3}$ Franklin Mendivil, ${ }^{4}$ \\ Manuel Ruiz Galan, ${ }^{5}$ and Rachad Zaki ${ }^{2}$ \\ ${ }^{1}$ Department of Economics, Management, and Quantitative Methods, University of Milan, 20122 Milan, Italy \\ ${ }^{2}$ Department of Applied Mathematics and Sciences, Khalifa University, P.O. Box 127788, Abu Dhabi, UAE \\ ${ }^{3}$ Department of Mathematics and Statistics, University of Guelph, Guelph, ON, Canada N1G 2W1 \\ ${ }^{4}$ Department of Mathematics and Statistics, Acadia University, Wolfville, NS, Canada B4P 2R6 \\ ${ }^{5}$ Department of Applied Mathematics, University of Granada, 18071 Granada, Spain
}

Correspondence should be addressed to Davide La Torre; davide.latorre@unimi.it

Received 27 September 2015; Accepted 28 September 2015

Copyright (C) 2015 Davide La Torre et al. This is an open access article distributed under the Creative Commons Attribution License, which permits unrestricted use, distribution, and reproduction in any medium, provided the original work is properly cited.

It is a real pleasure to announce the publication of this second special issue of this journal dedicated to highlighting recent research, development, and applications of inverse problems in science and engineering. This volume follows a similar special issue published in 2014 [1] and contains interesting and original papers.

In recent years a great deal of attention has been paid to the problem of parameter estimation in distributed systems, that is, the determination of unknown parameters in the functional form of the governing model of the phenomenon under study [2-7] from the observed data. In the mathematical literature, this kind of problem is called an inverse problem. According to Keller [8], "we call two problems inverse of one another if the formulation of each involves all or part of the solution of the other. Often, for historical reasons, one of the two problems has been studied extensively for some time, while the other one is newer and not so well understood. In such cases, the former is called the direct problem, while the latter is the inverse problem." However, there is an important and fundamental difference between the direct and the inverse problem: often the direct problem is well-posed while the corresponding inverse problem is ill-posed [9]. In the literature, one can find many contributions presenting ad hoc methods to address ill-posed inverse problems that involve the minimization of a suitable approximation error along with the use of some regularization techniques. In recent years, theory and applications of inverse problems have undergone tremendous growth: nowadays inverse problems can be formulated in many mathematical areas and analyzed by different theoretical and computational techniques.

In the paper "An Adaptive Observer-Based Algorithm for Solving Inverse Source Problem for the Wave Equation," by S. Asiri et al., the authors are interested in an inverse source problem for the wave equation. In particular, the objective of this paper is to present an alternative algorithm, based on observers, to solve the inverse source problem for the wave equation. Observers are well known in control theory. Originally designed to estimate the hidden states of dynamical systems given some measurements, the observers' scope has been recently extended to the estimation of some unknowns, for systems governed by partial differential equations. In this paper, observers are used to solve an inverse source problem for a one-dimensional wave equation. An adaptive observer is designed to estimate the state and source components for a fully discretized system. The effectiveness of the algorithm is emphasized in noise-free and noisy cases and an insight into the impact of measurements' size and location is provided.

The aim of the article "Approximate Image Reconstruction in Landscape Reflection Imaging," by R. Régnier et al., is to single out an imaging concept based on the phenomena of wave reflection on more or less opaque objects and register the reflected wave energy by a single detector. Reflection imaging of landscape (scenery or extended objects) poses the inverse problem of reconstructing the landscape reflectivity function from its integrals on some particular family of spheres. Such data acquisition is encoded in the framework 
of a Radon transform on this family of spheres. In spite of the existence of an exact inversion formula, the numerical landscape reflectivity function reconstitution is best obtained via an approximate but judiciously chosen reconstruction kernel. The authors describe the working of this reflection imaging modality and its theoretical handling, introduce an efficient and stable image reconstruction algorithm, and present simulation results to prove the validity of this choice as well as to demonstrate the feasibility of this imaging process.

A study on a detection method for continuous mechanical deformations of coaxial cylindrical waveguide boundaries using perturbation theory is discussed in the paper "Perturbation Approach to Reconstructing Deformations in a Coaxial Cylindrical Waveguide" by M. Dalarsson et al. The inner boundary of the waveguide is described as a continuous PEC structure with deformations modeled by suitable continuous functions. In this approach, the computation complexity is significantly reduced compared to discrete conductor models studied in previous works. If the mechanically deformed metallic structure is irradiated by the microwave fields of appropriate frequencies, then the authors can reconstruct the continuous deformation function by means of measurements of the scattered fields at both ends. They also apply the first-order perturbation method to the inverse problem of reconstruction of boundary deformations, using the dominant TEM-mode of the microwave radiation. Different orders of Tikhonov regularization, using the L-curve criterion, are investigated. Using reflection data, the authors obtain reconstruction results that indicate an agreement between the reconstructed and true continuous deformations of waveguide boundaries.

The article entitled "Nonlinear Inverse Problem for an Ion-Exchange Filter Model: Numerical Recovery of Parameters," by B. Mukanova and N. Glazyrina, considers the problem of identifying unknown parameters for a mathematical model of an ion-exchange filter via measurement at the outlet of the filter. The proposed mathematical model consists of a material balance equation, an equation describing the kinetics of ion-exchange for the nonequilibrium case, and an equation for the ion-exchange isotherm. The material balance equation includes a nonlinear term that depends on the kinetics of ion-exchange and several parameters. First, a numerical solution of the direct problem, the calculation of the impurities concentration at the outlet of the filter, is provided. Then, the inverse problem of finding the parameters of the ion-exchange process in nonequilibrium conditions is formulated. A method for determining the approximate values of these parameters from the impurities concentration measured at the outlet of the filter is proposed.

Since the geological bodies where tunnels are located have uncertain and complex characteristics, the inverse problem plays an important role in geotechnical engineering. In the paper "Back Analysis of Geomechanical Parameters Using Hybrid Algorithm Based on Difference Evolution and Extreme Learning Machine," by Z. Song et al., the authors propose a new hybrid algorithm that improves the accuracy and speed of surrounding rock identification, construct the back analysis objective function with usage of the displacement and stress monitoring data, and propose a hybrid algorithm. An extreme learning machine (ELM) is employed with optimal architecture trained by the difference evolution (DE) arithmetic. First, a three-dimensional numerical simulation is used in the creation of training and testing samples for ELM model construction. Second, the nonlinear relationship between rock parameters and displacement is constructed by numerical simulation. Finally, the geophysics parameters are obtained by DE optimization arithmetic taking into consideration the monitoring data that includes both displacement and pressure. This method had been applied in the Fusong highway tunnel in Fusong city of China's Jilin province, with a good effect obtained. It takes full advantage of DE and ELM and has both calculation speed and precision in the back analysis.

In the paper "Identification of Hydraulic Conductivity in Aquifer for Coupled FEM and Adaptive Genetic Algorithm," by X. Deng et al., the authors use hydraulic conductivity to conduct an inversion analysis according to the measurement of head materials by combining the finite element method with the adaptive genetic algorithm. The hydraulic conductivity of a natural rock mass is difficult to be determined because of the complex structure and the significant influence of uncertain factors. The results presented in this paper show that the maximum relative error of the measured and computed groundwater levels at the measuring points is $5.3 \%$ and the average head error is $1.41 \%$. The effective hydraulic conductivity of intensively weathered layer, moderately weathered layer, and fresh bedrock layer in riverbed formation tends to decline gradually and the effective permeability coefficient in the vertical direction is the minimum in the same aquifer. This analysis suggests then that the established hydraulic conductivity inversion analysis method is effective.

Finally, the paper "Joint Motion Deblurring and Superresolution from Single Blurry Image," by L. He et al., proposes a joint blind-deblurring and superresolution algorithm from one single image that combines gradient and motion blur kernel priors in a coherent framework. Currently, obtaining superresolution from a motion blurred image remains a challenging task. The conventional approach, which preprocesses the blurry low resolution (LR) image with a deblurring algorithm and employs a superresolution algorithm, has the limitation that the high frequency texture of the image is unavoidably lost in the deblurring process, and this loss restricts the performance of the subsequent superresolution process. This paper presents a novel technique that performs motion deblurring and superresolution jointly from one single blurry image. The basic idea is to regularize the ill-posed reconstruction problem using an edge-preserving gradient prior and a sparse kernel prior. This method derives from an inverse problem approach under an efficient optimization scheme that alternates between blur kernel estimation and superresolving until convergence. Furthermore, this paper proposes a simple and efficient refinement formulation to remove artifacts and render better deblurred high resolution (HR) images. The improvements brought by the proposed combined framework are demonstrated by the processing results of both simulated and real-life images. Quantitative 
and qualitative results on challenging examples show that the proposed method outperforms the existing methods and effectively eliminates motion blur and artifacts in the superresolved image.

\title{
Acknowledgments
}

The guest editors thank all of the authors as well as all others who submitted papers for consideration.

\author{
Davide La Torre \\ Herb Kunze \\ Franklin Mendivil \\ Manuel Ruiz Galan \\ Rachad Zaki
}

\section{References}

[1] H. E. Kunze, D. La Torre, F. Mendivil, M. Ruiz Galan, and R. Zaki, "Inverse problems: theory and application to science and engineering," Mathematical Problems in Engineering, vol. 2014, Article ID 497413, 2 pages, 2014.

[2] A. Kirsch, An Introduction to the Mathematical Theory of Inverse Problems, Springer, New York, NY, USA, 2011.

[3] H. Kunze, D. La Torre, F. Mendivil, and E. R. Vrscay, FractalBased Methods in Analysis, Springer, New York, NY, USA, 2012.

[4] F. D. Moura Neto and A. J. da Silva Neto, An Introduction to Inverse Problems with Applications, Springer, New York, NY, USA, 2013.

[5] A. Tarantola, Inverse Problem Theory and Methods for Model Parameter Estimation, SIAM, Philadelphia, Pa, USA, 2005.

[6] A. N. Tychonoff, "Solution of incorrectly formulated problems and the regularization method," Doklady Akademii Nauk SSSR, vol. 151, pp. 501-504, 1963.

[7] C. R. Vogel, Computational Methods for Inverse Problems, SIAM, Philadelphia, Pa, USA, 2002.

[8] J. B. Keller, "Inverse problems," The American Mathematical Monthly, vol. 83, no. 2, pp. 107-118, 1976.

[9] J. Hadamard, Lectures on the Cauchy Problem in Linear Partial Differential Equations, Yale University Press, 1923. 


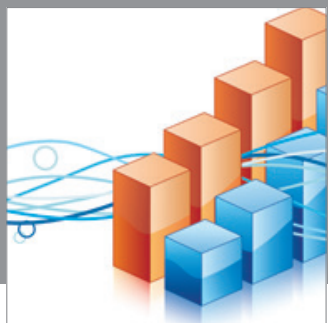

Advances in

Operations Research

mansans

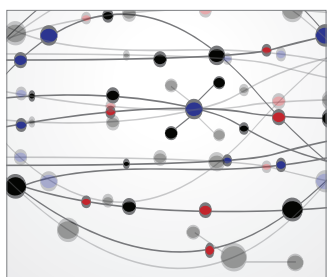

The Scientific World Journal
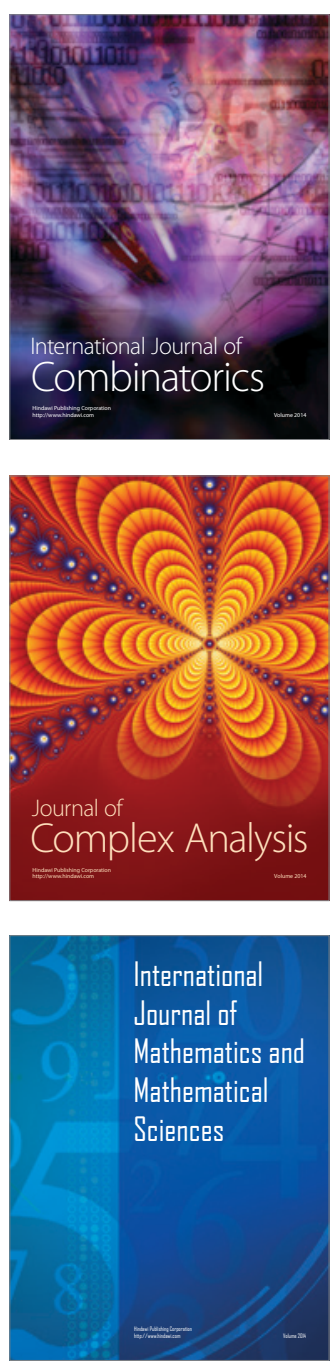
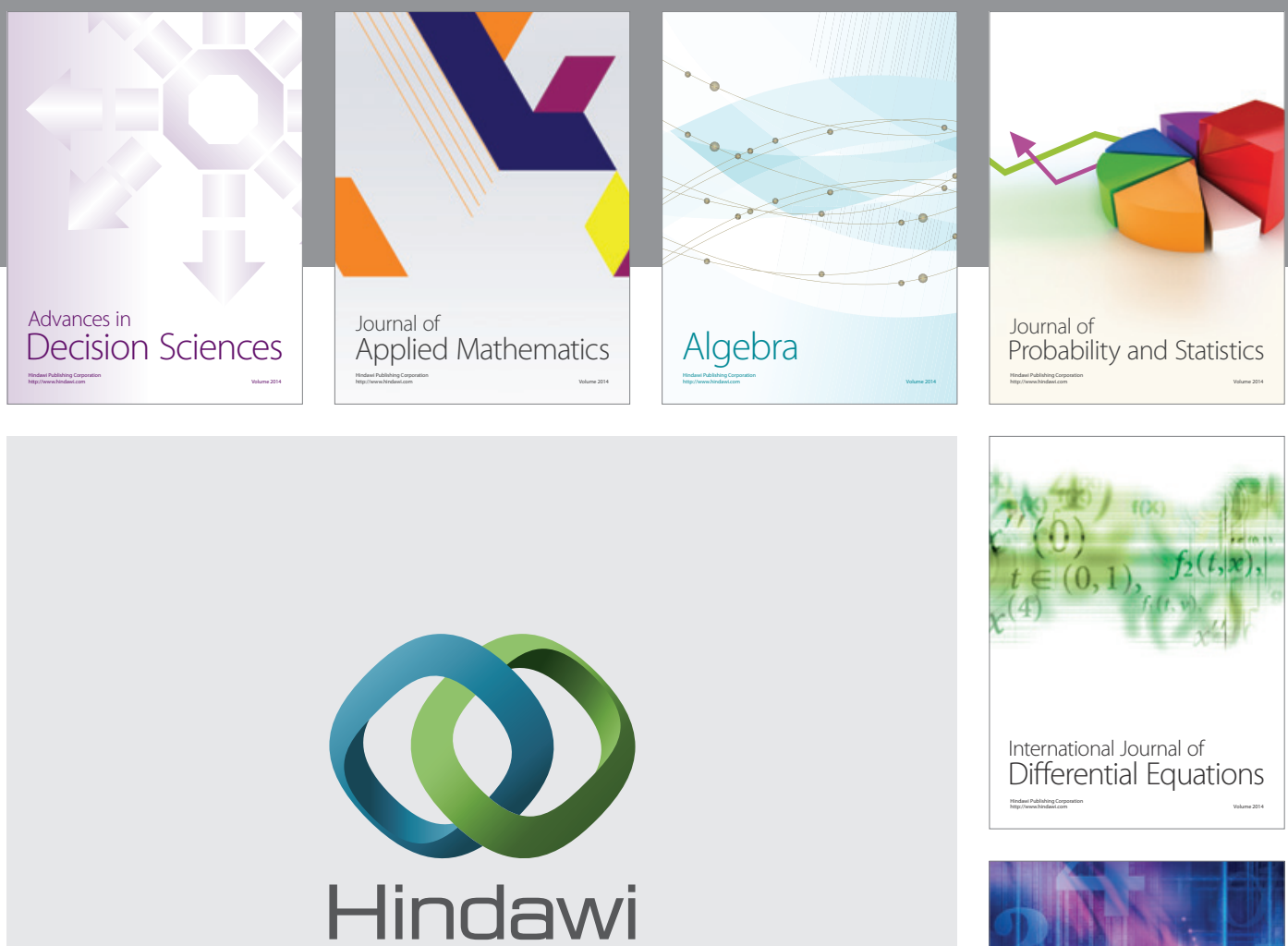

Submit your manuscripts at http://www.hindawi.com
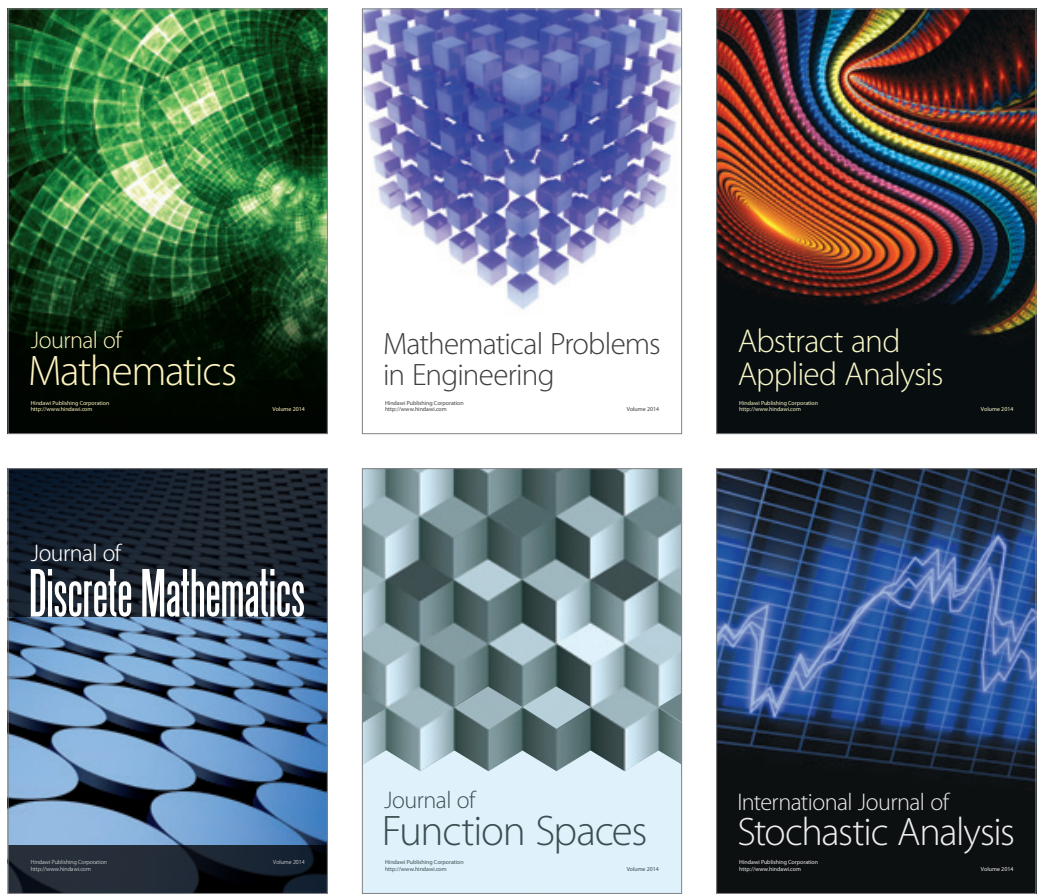

Journal of

Function Spaces

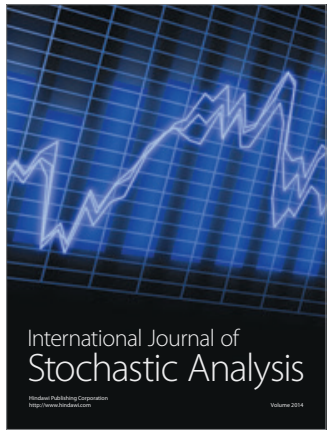

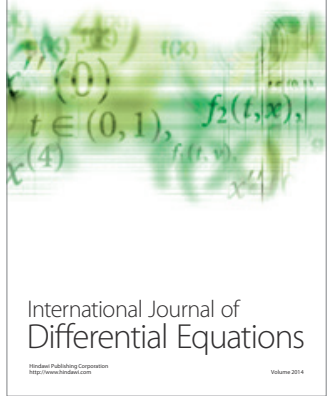
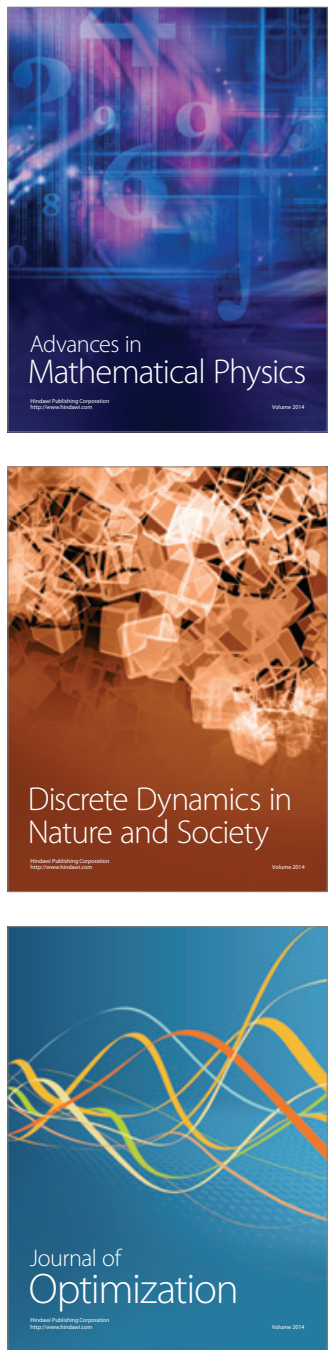